\title{
An Electrochemical Hydroquinone Sensor with Nitrogen-Doped Graphene Modified Electrode
}

\author{
Xueliang Niu, Lijun Yan ${ }^{1}$, Xiaobao $\mathrm{Li}^{1}$, Zuorui Wen ${ }^{1}$, Jianhua $\mathrm{Yu}^{2}$, Anhui Hu ${ }^{2}$, Lifeng Dong ${ }^{2,3 *}$, \\ Zaifeng Shi ${ }^{1}$,Wei Sun ${ }^{1 *}$ \\ ${ }^{1}$ College of Chemistry and Chemical Engineering, Key Laboratory of Water Pollution Treatment and \\ Resource Reuse of Hainan Province, Hainan Normal University, Haikou, Hainan 571158, P. R. China; \\ ${ }^{2}$ College of Materials Science and Engineering, Qingdao University of Science and Technology, \\ Qingdao 266042, P. R. China \\ ${ }^{3}$ Department of Physics, Hamline University, St. Paul, MN 55104, USA \\ *E-mail: swyy26@hotmail.com and Dong03@hamline.edu
}

doi: $10.20964 / 2016.08 .65$

Received: 10 May 2016 / Accepted: 19 June 2016 / Published: 7 July 2016

In this paper a solvethermal synthesized nitrogen-doped graphene (NG) was used as modifier on carbon ionic liquid electrode (CILE) to get a novel modified electrode, which was denoted as NG/CILE. An electrochemical hydroquinone (HQ) sensor was constructed with NG/CILE as working electrode. Electrochemical behaviors of HQ on NG/CILE were investigated by cyclic voltammetry with a pair of redox peaks observed. Compared with that of CILE, electrochemical responses of HQ were enhanced greatly with the peak-to-peak separation reduced. The result indicated good electrocatalytic ability of NG/CILE to the redox reaction of HQ. Under the optimal conditions a wider linear response between the peak current and HQ concentration was established in the concentration

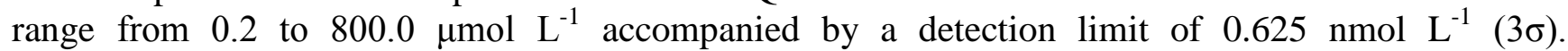
Furthermore, the as-proposed HQ sensor exhibited high sensitivity and good selectivity toward HQ detection, which was successful applied to the synthetic water samples analysis.

Keywords: Nitrogen-doped graphene; Carbon Ionic Liquid Electrode; Hydroquinone; Electrochemistry; Electrochemical sensor

\section{FULLTEXT}

(C) 2016 The Authors. Published by ESG (www.electrochemsci.org). This article is an open access article distributed under the terms and conditions of the Creative Commons Attribution license (http://creativecommons.org/licenses/by/4.0/). 